\title{
Alveolar bone trabeculae in patients with aggressive patients with aggressive periodontitis using cone beam computed tomography imaging
}

\author{
Shaliha*, Ria Noerianingsih Firman*, Yanti Rusyanti* \\ *Department of Dentomaxillofacial Radiology Faculty of Dentistry, Universitas Padjadjaran \\ ${ }^{* *}$ Department of Periodontics Faculty of Dentistry, Universitas Padjadjaran
}

\section{ABSTRACT}

Introduction: Periodontitis is an inflamatory process in supporting tissues of the teeth including the gingiva, cementum, periodontal ligament and alveolar bone. Perioditis ntitis begins with migration of junctional ephithelium toward the apical side and form a pocket on gingiva. Aggressive periodontitis is one of the classifications of periodontitis with characteristics of attacking teens to young adults in relatively fast can lead to severe alveolar bone loss and it is not comparable with local factors that there. Trabeculae are part of the alveolar bone covered by compact bone and cortical bone harder. On radiographs, trabeculae only seen as a radiolucent surrounded by a radiopaque. The purpose of this research was to know the description of alveolar trabecular bone in patients with aggressive periodontitis using Cone Beam Computed Tomography imaging. Methods: This study is descriptive on 72 samples of aggressive periodontitis alveolar travecular bon e by CBCT imaging, taken from 6 archival aggressive periodontitis patients as research subjects, obtained from secondary data in the Radiology Department of RSGM FKG UNPAD. Results: The results of this study from the 3D CBCT imaging of alveolar trabecular bone leading to further describe the more posterior a decline in trabecular bone density and decrease in patients with aggressive periodontitis. Conclusion: The 3D CBCT imaging of alveolar trabecular bone leading to further describe the more posterior a decline in trabecular bone density and decrease in patients with aggressive periodontitis.

Keywords: Aggressive periodontitits, trabeculae alveolar bone destruction, CBCT imaging.

\section{INTRODUCTION}

Periodontitis is an infection of the periodontal tissue including gums, cementum, periodontal ligament, and alveolar bone. This disease commonly occurs as a result of an inflammatory process that starts from the gums and continues to the deeper supporting tissues of the teeth, which can result in tooth loss. ${ }^{1}$ This disease is characterized by the formation of pockets in the gums resulting in gum recession and begins with the migration of the junctional epithelium to the apical, loss of attachment and alveolar bone crest. ${ }^{1}$ Clinical examination of the disease shows in increase of probing depth, bleeding on probing at the active site of the disease and changes in contour and physiological color of the gums, and usually it is painless. ${ }^{2}$

Corresponding author: Ria Noerianingsih Firman, Department of Dentomaxillofacial Radiology, Faculty of Dentistry Universitas Padjadjaran, Indonesia. Jalan Sekeloa Selatan I, Bandung, West Java, Indonesia, 40132; 
AAP International Workshop for Classification of Periodontal Disease, 1999 states that periodontal disease is divided into gingival disease, chronic periodontitis, aggressive periodontitis, periodontitis as manifestations of systemic disease, necrotic periodontal disease, periodontal abscess, periodontitis associated with endodontic lesions, deformity and state of solids bone. $^{1}$

Aggressive periodontitis is a periodontal tissue infection that usually attacks adolescents and young adults. In general, it looks healthy and has the characteristics of fast alveolar bone loss in more than one tooth, especially incisors and molar teeth with severity that occurs not in accordance with existing local factors.

Patients often have significant colonization of bial Actinobacillus actinomycetemcomitans. Two-dimensional radiographs of localized aggressive periodontitis include arch-shaped alveolar bone loss extending from the distal surface of the second premolar to the mesial surface of the second molar. Two-dimensional radiographs of generalized aggressive periodontitis show widespread bone loss in most teeth including molars and incisors. ${ }^{3.1}$

Alveolar bone resorption associated with periodontal disease occurs on all surfaces of the tooth and can be seen on radiographic examination. Normally, the alveolar crest is $1-2$ $\mathrm{mm}$ in the apical direction of the cemento-enamel junction. If there is bone loss, the alveolar crest is more than $2 \mathrm{~mm}$ in the apical direction of the cemento-enamel junction. ${ }^{4}$

The alveolar bone is the jawbone that supports the teeth. These bones are made up of cancellous and dense bones, layered by cortical bone. The presence of alveolar bone depends on the state of the teeth. After missing teeth, the alveolar bone will undergo resorption..$^{5,6}$ The height and thickness of the alveolar bone on the facial or buccal and lingual or palatinal surfaces is influenced by the shape and arrangement of the teeth and is influenced by external pressure. The alveolar bone undergoes continuous reconstruction, adapts to functional requirements and is influenced by systemic factors. Alveolar bone consists of an outer cortical bone layer, an inner cortical bone layer of the tooth socket wall, and Cancellous trabeculae. ${ }^{1}$
The trabeculae are part of the alveolar bone with a spongy, hollow structure. Trabeculae have a higher metabolism than cortical bone, so they become more brittle and break easily, but they provide maximum support even with a minimum amount of bone material. ${ }^{7}$ Trabecular features on conventional radiographs show thin radiopaque and surrounded by radiolucent. ${ }^{8}$

Radiographs are an important tool in diagnosing periodontal disease. Radiographs show alveolar bone height and width, bone damage patterns, periodontal membrane space width, and cancellous trabeculation. ${ }^{6.1}$ Detection of periodontal disease can be carried out with radiological diagnostic tools, so to compare the diagnostic accuracy of conventional radiographs (periapical radiographs) and digital (zonography), several independent observers assess the results of radiographs in detail on widening periodontal ligament, alveolar crest resorption, vertical bone loss, furcation involvement and the presence of calculus. The results of the analysis conclude that the sensitivity of zonography radiographs showed a more detailed periapical picture in detecting periodontal disease. ${ }^{8}$ Currently, alveolar bone trabeculae can be seen more clearly, while getting the right composition between radiopaque and radiolucent images usingCone Beam Computed Tomography (CBCT). CBCT is a radiographic device that utilizes conical $\mathrm{x}$-rays and computerized volumetric reconstructions that produce threedimensional radiographs that are sagittal, coronal, and axial. Radiographs produced by this CBCT tool can interpret various things needed in the evaluation process, such as showing a histogram, radiographs of specific parts desired by the doctor or patient, measuring the distance of an area, density, surface image, modulation intensity, osseointegration, etc. ${ }^{9,10}$

Trabecular features on two-dimensional radiographs appear in thin radiopaque and surrounded by radiolucent. This does not provide clear and detailed picture in assisting enforcement stage of diagnosis. ${ }^{8}$ In addition, these two-dimensional radiographs are very limited as subjects for alveolar bone trabecular research. Advance of technology by using CBCT tool show a clearer and more detailed periodontal tissue. Aim of this research is to find out the picture of alveolar trabeculae in patients with aggressive 
periodontitis using Cone Beam Computed Tomography imaging.

\section{METHODS}

Type of research used is descriptive research, which is a research method that studies problems in society, procedures that apply in society and situations, attitudes, views, ongoing processes, the influence of a phenomenon; careful measurement of phenomena in society. Researchers develop concepts, gather facts, but do not test hypotheses. The population of this study was all radiographic data of patients with diagnoses of aggressive and chronic periodontitis in Faculty of Dentistry, Padjadjaran University, Bandung. The population of this study was the 3D Cone Beam Computed Tomography (CBCT) radiograph of aggressive periodontitis patients who came to Faculty of Dentistry of Padjadjaran University Bandung during the period May - July 2010.

Samples are secondary data. In total, $6 C B C T$ radiographic archives were obtained and 72 samples of alveolar bone trabeculae were obtained in patients with aggressive periodontitis.

The study was conducted at the Radiology department of the Dental and Oral Hospital of the Faculty of Dentistry, Padjadjaran University, Bandung in July 2011. The tools used in this study are data on 3D CBCT Radiographic CDs of aggressive periodontitis patients and the EazyDent program.

\section{RESULTS}

The study was conducted in July 2011 at Dental Radiology Department of the Dental and Oral Hospital of Padjadjaran University in Bandung, aiming to determine the picture of alveolar trabeculae in patients with aggressive periodontitis based on radiography using $\mathrm{CBCT}$ (Cone Beam
Computed Tomography) measurements. Samples obtained were 6 archives, including 3 archives of localized aggressive periodontitis and 3 archives of generalized aggressive periodontitis. So, 72 samples were obtained as a result of Cone Beam Computed Tomography imaging.

The results of this study are data in the form of radiographic percentage of alveolar trabeculae of patients with localized and generalized aggressive periodontitis which were seen using CBCT measurements. CBCT ( Cone Beam Computed Tomography ) measurements divide the trabecular pattern into a color ratio that can determine the percentage of radiopaque and radiolucent on alveolar bone trabecular radiography. The orange color represents the percentage of radiolucent and the red color represents the percentage of radiopaque. The following table shows the results of measurements for regions I1 (Incisive 1) and M1 (Molar 1) of the upper and lower jaw in patients with localized aggressive periodontitis and regions 12 (Incisive 2), C (Canine), P1 (Premolar 1), P2 (Premolar 2), and M2 (Molar 2) of the upper and lower jaw in generalized aggressive periodontitis patients.

Table 1 shows the percentage of radiographic images of maxillary alveolar trabeculae for periodontitis aggressive localized, and it can be seen that the maxillary trabecular region of 11 radiopaque is higher, $90.5 \%$ compared to the percentage of maxillary $M 1$ trabecular radiopaque region at $30.51 \%$. The percentage of radiolucent on maxillary I1 trabecular region was lower at 9.5\% compared to the percentage of radiolucents of the maxillary trabecular region of $69.49 \%$.

Figure 1 shows the difference in the percentage of radiopaque and radiolucent images of maxillary I1 and $M 1$ teeth. $I 1$ tooth radiopaque percentage is higher than $M 1$ tooth; conversely percentage of radiolucent 11 tooth is lower than M1

Table 1. Percentage measurement results (radiopaque and radiolucent) trabeculae incisive region 1 and maxillary molar 1 in local aggressive periodontitis

\begin{tabular}{ccccccc}
\hline \multirow{2}{*}{ Region } & \multirow{2}{*}{ Overview (\%) } & \multicolumn{3}{c}{ Sample } & Amount & Average \\
\cline { 3 - 7 } & & 1 & 2 & 3 & & \\
\hline I1 & Radiopaque & 99,215 & 96.38 & 75,895 & 271.49 & 90.5 \\
M1 & & 20,405 & 26,195 & 44.93 & 91.53 & 30.51 \\
I1 & \multirow{2}{*}{ Radiolucent } & 0.785 & 3.62 & 24,105 & 28.51 & 9.5 \\
M1 & & 79,595 & 73,805 & 55.07 & 208.47 & 69.49 \\
\hline
\end{tabular}




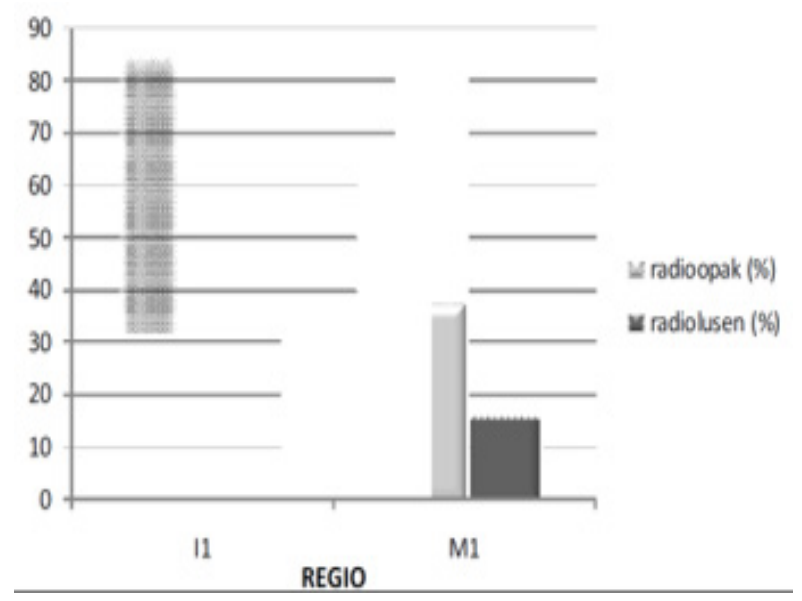

Figure 1 . Percentage of images (radiopaque and radiolucent) trabecular incisive region 1 and maxillary molar 1 in aggressive periodontitis

Table 2 . Percentage (radiopaque and radiolucent) percentage measurement results of incisive region 1 and lower molar 1 trabeculae in aggressive periodontitis

\begin{tabular}{|c|c|c|c|c|c|c|}
\hline \multirow{2}{*}{ Region } & \multirow{2}{*}{ Overview (\%) } & \multicolumn{3}{|c|}{ Sample } & \multirow[t]{2}{*}{ Amount } & \multirow[t]{2}{*}{ Average } \\
\hline & & 1 & 2 & 3 & & \\
\hline 11 & Radiopaque & 66 & 91.59 & 94.18 & 251.77 & 83.92 \\
\hline M1 & & 34.87 & 13,615 & 63.63 & 112,115 & 37.37 \\
\hline 11 & Radiolucent & 34 & 8.41 & 5.82 & 48.23 & 16.07 \\
\hline M1 & & 65.13 & 86.385 & 36.37 & 187.885 & 62.63 \\
\hline
\end{tabular}

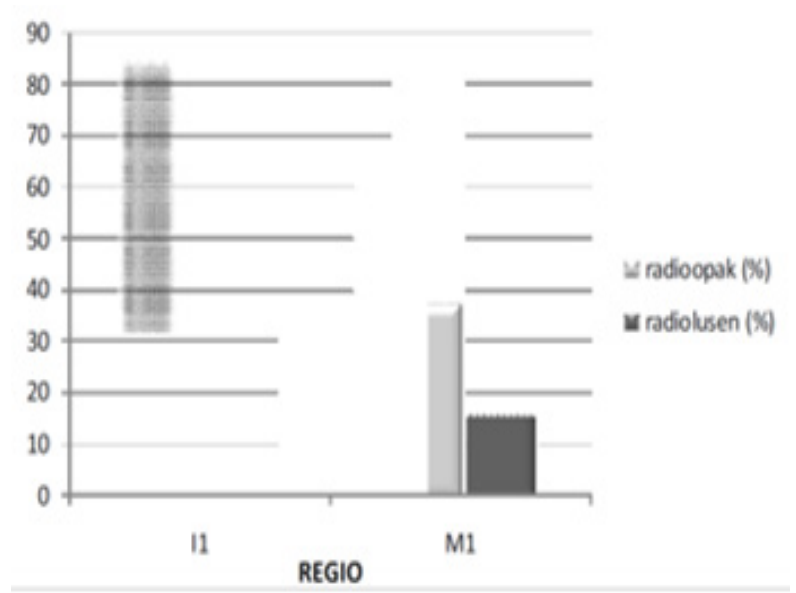

Figure 2 . Percentage of images (radiopaque and radiolucent) trabeculae incisive region 1 and lower molar 1 in aggressive periodontitis

tooth. This shows that the percentage of anterior tooth bone density is higher than that of posterior teeth with localized aggressive periodontitis.

The measurement of the percentage of radiographic images of alveolar trabeculae in generalized periodontitis of the lower jaw is shown in Table 4.2 , the table shows that the radiopaque percentage of the mandibular 11 trabecular region is higher, which is $83.92 \%$ compared to the radial percentage of the mandibular M1 trabeculae of $37.37 \%$. The percentage of radiolucent in the lower jaw 11 region of trabeculae was lower at $16.07 \%$ compared to the percentage of radiolucent in the lower jaw M1 trabeculae of $62.63 \%$.

Table 2 gives the same conclusion as the previous table, the percentage of anterior radiopaque teeth is higher than the percentage of posterior radiopaque teeth, and vice versa with the 
percentage of radiolucent. Based on Figure 2, it can be seen the difference in percentage of alveolar bone trabeculae in lower jaw 11 and M1 teeth.

Table 3 and 4 shows the percentage measurements of radiographs of the maxillary and mandibular alveolar trabeculae in generalized aggressive periodontitis. Based on table 3 , the results showed that the highest radiopaque percentage of $79.5 \%$ was in the upper jaw region trabecula 12 , while the lowest radiopaque trabeculae percentage was in the maxillary $M 2$ region of $33.64 \%$. While the highest radiolucent percentage is in the maxillary $M 2$ trabeculae at $66.35 \%$ and the lowest radiolucent percentage is in the maxillary 12 trabeculae at $20.5 \%$.

Figure 3 shows the trabeculae of regions 12 to $M 2$ of the maxilla which had a greater percentage of radiolucent in the posterior and a smaller percentage of radiopaque in the posterior.
Table 4 gives the radiographic percentages of alveolar trabeculae in generalize aggressive periodontitis of mandibular. Based on Table 4, the highest radiopaque percentage was $100 \%$ in trabecula 12 of the lower jaw region, while the lowest radiopaque percentage was in the lower jaw $M 2$ region by $44.5 \%$. While the highest radiolucent percentage is in the lower jaw M2 trabeculae at $55.5 \%$ and the lowest radiolucent percentage is in the lower jaw trabeculae of $0 \%$.

From the picture, trabeculae of regions 12 to $M 2$ of the mandibular shows radiolucent ratio increases posteriorly and radiopaque comparison decreases posteriorly. This supports the greater percentage of radiopaque in the anterior teeth and decreases regularly in the posterior teeth. Conversely, the more posterior position of the teeth, the greater the percentage of radiolucent.

Table 3 . Percentage measurement results (radiopaque and radiolucent) trabeculae incisive region 2, canine, premolar 1 , premolar 2 and upper molar 2 in aggressive periodontitis

\begin{tabular}{|c|c|c|c|c|c|c|}
\hline \multirow{2}{*}{ Region } & \multirow{2}{*}{ Overview (\%) } & \multicolumn{3}{|c|}{ Sample } & \multirow[t]{2}{*}{ Amount } & \multirow[t]{2}{*}{ Average } \\
\hline & & 4 & 5 & 6 & & \\
\hline 12 & & 100 & 56 & 82.5 & 238.5 & 79.5 \\
\hline C & & 100 & 74.8 & 55.45 & 230.25 & 76,75 \\
\hline Q1 & Radiopaque & 97,415 & 54.61 & 44.58 & 196,605 & 65,535 \\
\hline P2 & & 68.93 & 78.08 & 48.88 & 195.89 & 65.29 \\
\hline$M 2$ & & 97,765 & 0 & 3,165 & 100.93 & 33.64 \\
\hline 12 & & 0 & 44 & 17.5 & 61.5 & 20.5 \\
\hline C & & 0 & 25.2 & 44.55 & 69.75 & 23.25 \\
\hline Q1 & Radiolucent & 2,585 & 45.39 & 55.42 & 103,395 & 34,465 \\
\hline P2 & & 31.07 & 21.92 & 51.12 & 104.11 & 34,703 \\
\hline M2 & & 2,235 & 100 & 96,835 & 199.07 & 66,356 \\
\hline
\end{tabular}

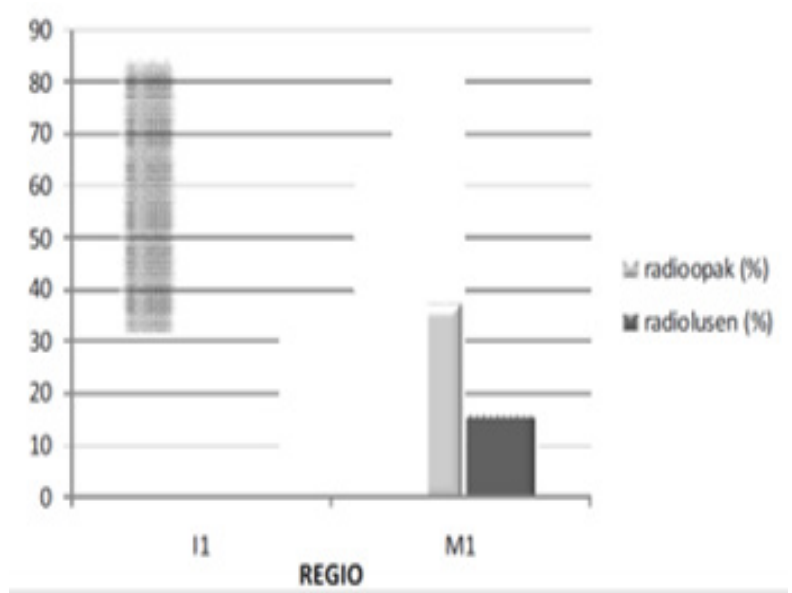

Figure 3 . Percentage of images (radiopaque and radiolucent) trabeculae incisive region 2, canine, premolar 1, premolar 2 and upper molar 2 in generalize aggressive periodontitis 
Table 4 . Percentage measurement results (radiopaque and radiolucent) trabeculae region incisive 2, canines, premolar 1, premolar 2 and lower molar 2 in aggressive periodontitis

\begin{tabular}{ccccccc}
\hline \multirow{2}{*}{ Region } & Overview (\%) & \multicolumn{3}{c}{ Sample } & Amount & Average \\
\cline { 3 - 6 } & & 4 & 5 & 6 & & \\
\hline I2 & 100 & 100 & 100 & 300 & 100 \\
C & 98.91 & 84.45 & 96.15 & 279,51 & 93.17 \\
Q1 & Radiopaque & 89,725 & 40.85 & 57.505 & 188.08 & 62.69 \\
P2 & & 52.35 & 86.03 & 48,135 & 186,515 & 62.17 \\
M2 & 70,765 & 20.2 & 42,525 & 133.49 & 44.5 \\
I2 & 0 & 0 & 0 & 0 & 0 \\
C & & 1.09 & 15.55 & 3.85 & 20.49 & 6.83 \\
Q1 & & 10,275 & 59,15 & 42,495 & 111.92 & 37,306 \\
P2 & & 47.65 & 13.97 & 51,865 & 113,485 & 37.82 \\
M2 & & 29,235 & 79.8 & 57,475 & 166.51 & 55.5 \\
\hline
\end{tabular}

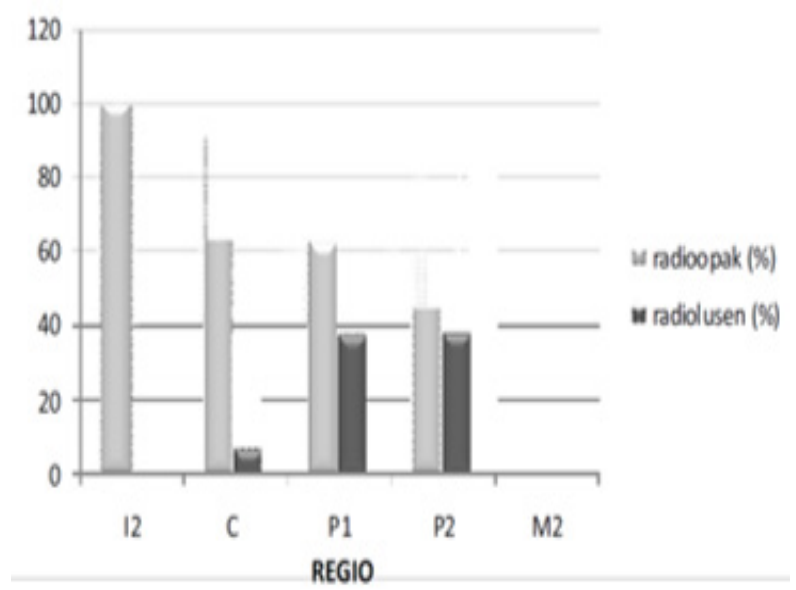

Figure 4 . Percentage of images (radiopaque and radiolucent) trabeculae incisive region 2, canine, premolar 1, premolar 2 and lower molar 2 in generalized aggressive periodontitis

\section{DISCUSSION}

Periodontitis is an infection of the periodontal tissue including gums, cementum, periodontal ligament, and alveolar bone. This disease commonly occurs as a result of an inflammatory process that starts from the gums and continues to the deeper supporting tissues of the teeth, which can lead to tooth loss.

Changes that occur in alveolar bone on periodontitis patients are very important because boneloss cancause toothloss. Theheightand density of alveolar bone under normal circumstances have a balance between the magnitude of formation and resorption which is regulated by systemic factors and local factors. When the value of resorption is greater than the value of bone formation, alveolar bone height and density can decrease.
The main cause of bone damage in periodontal disease is the spreading of inflammation of gingival marginal to the supporting tissues. The invasion of gingival inflammation to the bone surface and the onset of bone loss are the main characteristics of the transition from gingivitis to periodontitis. Periodontitis is always preceded by gingivitis, whereas not all gingivitis develops into periodontitis. Factors that cause the spreading of inflammation into the supporting tissues and initiate the change of gingivitis into periodontitis are associated with the bacterial composition found in plaque. In severe periodontal disease, the content of movable bacteria and spirochaeta increases while coccus and stem bacteria decrease.

The spreading of inflammation is also associated with the pathogenic potential of 
plaque, host resistance, including human immunological reactions, and tissue reactions such as the degree of gingival fibrosis, area of attached gingiva , fibrogenesis and reactive osteogenesis. The fibrinolytic system is referred to as walling off from increased lesions.

Gingival inflammation extends along the bundle of collagen fibers and spreads along the blood vessel pathway to the alveolar bone. In the molar region, inflammation can extend to the maxillary sinus and cause thickening of the mucous sinus. In the interproximal area, inflammation spreads to the loose connective tissue around the blood vessels through fibers, spreads to the bone through the vessels and then perforates the crest of the interdental septum in the middle of the alveolar crest, then spreads to the sides of the interdental septum. In the facial and lingual, gingival inflammation spreads through the outer periosteal layer of bone and penetrates through blood vessels. After inflammation reaches the bone, inflammation spreads into an empty area and fills it with leukocytes, exudate fluid, new blood vessels, and proliferating fibroblasts. The number of multinuclear osteoclasts and phagocytosis increases, then the bone layer disappears and replaced with lacuna.

Factors that influence bone damage in periodontal disease are bacteria and hosts. Plaque bacterial products increase the differentiation of bone progenitor cells into osteoclasts and stimulate the gingival cells to release a mediator that triggers this. Plaque products and inflammatory mediators inhibit the work of osteoblasts and reduce the number of these cells, so that bone resorption activity increases, while the process of bone formation is inhibited so that bone loss occurs. ${ }^{1}$

Based on Figures 1 and 2, patients with localized aggressive periodontitis can be seen that the highest percentage of alveolar trabecular radiopaque $90.5 \%$ is in the maxillary incisive region, while the lowest radiopaque $30.51 \%$ is in the maxillary molar one region. The highest radiolucent percentage is $64.69 \%$ in the maxillary first molar region, and the lowest radiolucent percentage is $9.5 \%$ in the maxillary incisor region.

Based on Figure 3 and 4 in patients with generalized aggressive periodontitis, it can be seen that the highest percentage of alveolar bone trabecular radiopaque is $100 \%$ in the lower mandibular incisor region, while the lowest radiopaque percentage is $33.64 \%$ in the upper second molar region. The highest radiolucent percentage is $66.35 \%$ in the upper second molar region, and the lowest radiolucent percentage is $0 \%$ in the lower mandibular incisor region.

Based on figures 3 and 4 in the premolar region, it was found that the percentage of radiopaque and percentage of radiolucent had not much different results. The percentage of radiopaque of the maxillary first premolar region was $65.53 \%$ and the second premolar region was $65.29 \%$, while the percentage of radiolucent in the premolar region was $34.46 \%$ and in the second premolar region was $34.7 \%$. The percentage of radiopaque of the mandibular premolar region was $62.69 \%$ and the second premolar region was $62.17 \%$, while the radiolucent percentage of the premolar region was $37.3 \%$ and in the second premolar region was $37.82 \%$.

Based on the pictures above, it can be seen in sequence from the anterior to the posterior region, both in localized aggressive periodontitis and generalized aggressive periodontitis, the percentage of radiopaque decreases and the percentage of radiolucent increases. According to the principle of radiographic interpretation, radiopaque is a picture of bone whose composition contains the most calcium while opposite for radiolucent. ${ }^{11}$ Therefore, the results of this study indicate that the more posteriorly it is, the lower the bone density is. Alveolar bone resorption is more often found in the posterior region twice as much as in the anterior region. Functional activity is greater in the posterior teeth than in the anterior teeth, thus resulting in a posterior portion allowing a more severe decrease in bone density. If a force within physiological limits is applied to the bone, then the force, whether it is pressure or strain, can affect bone remodeling. ${ }^{12}$

Functional factors including the frequency intensity, duration, and direction of the force applied to the bone can be realized in the biological activity of the cell, resulting in bone formation or bone resorption, depending on the individual's resistance to the force. ${ }^{12}$

The presence of specific microflora species of A.actinomycetemcomitans is the main etiology of aggressive periodontitis and the presence of 
immune abnormalities and bad habits will further support bone resorption. The cell activity causes metabolic factors to be important because cell activity is influenced by the force that causes functional factors to be important. ${ }^{12}$

$$
\text { Some specific microorganisms }
$$

are often detected in PAL patients (A.actinomycetemcomitans, Capnocytophaga spp., Eikenella corrodens, Prevotella intermedia and Campylobacter rectus).

A.actinomycetemcomitans are mentioned as primary pathogens, as concluded by Tonetti and Mombelli: A.actinomycetemcomitans are found in high numbers on characteristics of the lesions from localized aggressive periodontitis (about 90\%), with evidence of lesion progression often showing elevated level $A$ .actinomycetemcomitans. Some patients with clinical manifestations of localized aggressive periodontitis have significantly increased serum antibodies to A.actinomycetemcomitans, and there is a relationship between subgingival burden reduction by A.actinomycetemcomitans during treatment and the success of clinical responses, A.actinomycetemcomitans produces a number of virulent factors that are subgingival in a subgingival load reduction by $A$. actinomycetemcomitans during treatment and the success of clinical responses can have an influence on the disease process.

Some immune damage has a relationship with the pathogenesis of periodontitis. Human leukocyte antigens (HLAs), which regulate immune responses, have been considered as a sign for aggressive periodontitis. Although HLAs are inconsistent, HLA and B15 antigens are consistently associated with aggressive periodontitis.

Some investigations show that patients with aggressive periodontitis have functional damage to polymorphonuclear leukocytes (PMNs), monocytes, or both. This damage can be weakened by the chemotaxis activity of PMNs at infected sites or by the ability of phagocytes to kill organisms. Current research has also shown a monocyte hypersensitivity from localized aggressive periodontitis patients that involves the production of their prostaglandin E2 (PGE2) when responding to lipopolysaccharide (LPS). This hyper responsive phenotype can lead to increased loss of connective tissue and bone caused by excessive production of catabolic factors. The immune system has an important role in systemic aggressive periodontitis. Anusaksathien and Dolby whom found antibodies to the host are collagen, deoxiribonucleic acid (DNA) and IgG. Immune mechanisms that may increase activity of major histocompatibility complex (MHC) class II molecules, HLA, DR4, T cell function suppressors, activation of polyclonal $B$ cells are plaque microbes and genetic predisposition.

Specifically, geneticfactorshaveimplications in aggressive periodontitis. Immunological damage associated with aggressive periodontitis can be genetically inherited. The results also showed that antibody responses to periodontal pathogens, including $A$. actinomycetemcomitans under genetic control. The amount of protective antibodies (especially lgG2) depends on race.

The amount and duration of smoking are important variables that influence the damage in young adults. Generalized aggressive periodontitis patients whom have the habit of smoking have more teeth affected and greater loss of clinical attachment than patients with generalized aggressive periodontitis who do not smoke. ${ }^{1}$

Based on graph 4.4 it was found that the radiopaque percentage was $100 \%$ and the radiolucent percentage was $0 \%$ in the mandibular incisor region, in contrast to the maxillary incisor region. In addition, overall results of the study show that bone resorption is identified by a greater percentage of radiolucent occurs in the maxilla compared to resorption that occurs in the lower jaw alveolar trabeculae.

Generally, the maxillary teeth have a downward and outward direction of inclination, so the reduction of the bone generally also occurs upward and inward, therefore the cortical plate of the outer bone is thinner than the inside. The outer cortical plate resorption goes more and faster. Inclination of the lower anterior teeth is generally upward and forwards from the occlusal plane, whereas the posterior teeth are more vertical or slightly tilted lingually. The outer surface of the bone cortical plate is thicker than the lingual surface, except in the molar region; the lower edge of the mandible is the thickest cortical layer. ${ }^{13}$ The results of the study explain that bone resorption occurs more in posterior teeth than in anterior teeth. Between the anterior maxilla and 
mandible, more resorption occurs in the maxillary anterior dentition.

Many factors can influence the results of this study, in addition to microflora, immunological, genetic, and environmental factors, the patient's age, poor lifestyle such as smoking and drinking soft drinks, the use of drugs such as glucocorticoids, and systemic diseases also affect the state of the trabecular bone. Nutrient intake can also play an important role in the alveolar bone trabecular density value. Poor nutritional intake can lead to nutritional disorders. Deficiency of certain substances needed in bone formation can affect the density of alveolar trabecular. ${ }^{14}$

There are deficiencies in the results of this study, and it is likely due to the weakness of the research results, one of which is the lack of the number of samples used in this study. Larger samples will provide more accurate results, but require more time, energy, money, increase the scope of research, and other facilities. ${ }^{15}$

\section{CONCLUSION}

The 3D CBCT imaging of alveolar trabecularboneleading to further describe the more posterior a decline in trabecular bone density and decrease in patients with aggressive periodontitis.

\section{REFERENCES}

1. Carranza FA, Newman MG. Clinical Periodontology. $10^{\text {th }}$ ed. London: Philadelphia WB. Saunders. Co., 2006. p. 452-66,50612,561-580.

2. Fedi PF, Vernino AR, Gray JL. The periodontic Syllabus. $4^{\text {th }}$ ed. Wolter Kluwer Company. 2004. p. 75-85.

3. Rose LF, Genco RJ. Periodontal Medicine. London: St. Louis: BC Decker, Inc. 2004.

4. Muller HP, Muller RF, Lange DE. Morphological compositions of subgingival microbiota in Actinobacillus actinomycetemcomitansassociated periodontitis. J Clin Periodontol 1979;17:549-56.

5. Hoag PM, Pawlak EA. Essentials of Periodontics. $4^{\text {th }}$ ed. St. Louis, Baltimore, Philadelphia, Toronto: CV Mosby Co. 1990. p. 13-17, 69-80.

6. Manson JD, Eley BM. Outline of Periodontics. $3^{\text {rd }}$ ed. London: Wright. 1993.

7. Van Resburg, Jansen BG. Oral Biology. Berlin: Quinstessence Publishing Co., Inc. 1995. p. 58-60, 87-8, 393-5.

8. Verlag Springer. Periodotal Manifestations of Local and Systemic Diseases. Copenhagen: Munkksgaard. 2003.

9. Araki K, Maki K, Seki K, Sakamaki K, Harata $Y$, Sakaino R et al. Characteristics of a newly developed dentomaxillofacial X-ray beam CT scanner (CB MercuRay ${ }^{\top M}$ ): system configuration and physical properties. Dentomaxillofac Radiol. 2004 Jan;33(1):51-9.

10. Goaz PW, White SC. Oral Radiology: Principles and Interpretation. $7^{\text {th }}$ ed. St. Louis: Mosby Co. 2004. p. 276-280.

11. Margono G. Guidelines for Making Intra Oral Radiograms. Special edition of Dentistry Magazine FORIL VI USAKTI 2006;2:200-6.

12. Atwood DA. Some clinical factors to rate the resorption of residual ridges. J Prosthet Dent. 2001;86(2):119-25.

13. Boucher TO, Elsayed, Elsayed A. Analysis and Control of production systems. $2^{\text {nd }}$ ed $\mathrm{New}$ Jersey: Prentice-Hall, Inc., Englewood Cliffs. 1982.

14. Ibeth AS. Overview of Mandibular Trabeculae in Edentulous Female Patients Aged 49-83 Years Based on CBCT Measurement Techniques. Bandung: Padjadjaran University. Thesis. 2011.

15. Notoatmodjo S. Health Research Methodology. Jakarta: Rineka Cipta. 2005. p. 89. 